\title{
ESTRATÉGIAS SOCIORRETÓRICAS PARA PROPOSIÇÃO DE PESQUISA NA CULTURA DISCIPLINAR DE QUÍMICA: UMA ANÁLISE DE PROJETOS DE PESQUISA DE EXPERTS
}

\author{
SOCIALRHETORICAL STRATEGIES IN RESEARCH PROPOSALS IN THE \\ CHEMISTRY DISCIPLINARY CULTURE: AN ANALYSIS OF PROPOSALS \\ WRITTEN BY EXPERIENCED RESEARCHERS
}

\author{
Francisco Alves Filho \\ Universidade Federal do Piauí, Teresina, Piauí, Brasil \\ chicofilhoo@ufpi.edu.br \\ Carolina Aurea Cunha Rio Lima \\ Universidade Federal do Piauí, Teresina, Piauí, Brasil \\ carolriolima@gmail.com
}

Resum0: Neste artigo, propomos uma análise dos passos retóricos "Relatando pesquisa prévia", "Indicando lacuna de pesquisa" e "Indicando problemas do mundo real" em projetos de pesquisa escritos por pesquisadores experientes da área de Química, buscando compreender sua relação com o estabelecimento da relevância da pesquisa. Os resultados mostram que o relato de pesquisas prévias é uma estratégia bastante importante e que permite contextualizar o projeto dentro das discussões e dos procedimentos já realizados anteriormente. As ocorrências da indicação de lacuna revelam a ênfase dada aos procedimentos metodológicos que devem ser modificados, e à insuficiência de pesquisas sobre determinados tópicos. Por fim, a indicação de problemas do mundo real evidencia o fato de que as pesquisas devem se preocupar em resolver problemas práticos do mundo, aliando o mundo acadêmico ao mundo social.

Palavras-chave: Projeto de pesquisa; Química; Pesquisa prévia; Lacuna de pesquisa; Problemas do mundo real

Abstract: In this article we propose an analysis of the rhetorical steps "Reporting preview research", "Indicating a research gap" and "Indicating problems in the real world" in research proposals written by experienced researchers in the Chemistry Field, seeking to understand their relation with the establishment of the relevance of the research. The results show that the report of preview research is a very important strategy and allows to contextualize the proposal inside the discussions and procedures previously performed. The occurrences of a research gap indicate the emphasis given to the methodological procedures that should be modified, and to the insufficiency of researches about some topics. Lastly, the indication of problems in the real world highlights that researches should be able to resolve practical problems in the world, uniting the academic and social worlds.

Keywords: Research proposal; Chemistry; Preview research; Research gap; Problems in the real world 


\section{INTRODUÇÃO}

Esta pesquisa se insere em uma perspectiva sociorretórica, entendida em sentido amplo e compreendendo esse termo dentro do que propõem Meurer, Bonini e Motta-Roth (2005). Assim, partimos de uma noção de gênero fundamentada em Miller (2012 [1984]) e em Swales (1990), os quais compreendem os gêneros como inerentemente sociais, pois as diferentes comunidades discursivas (SWALES, 1990, 2016) se apropriam dos gêneros de maneiras distintas, e como caracteristicamente retóricos, haja vista realizarem os propósitos comunicativos das comunidades discursivas (SWALES, 1990). O aspecto social, aqui, reside em analisar textos de uma comunidade particular de pesquisadores da área de Química. $\mathrm{O}$ aspecto retórico reside na compreensão de que as estratégias retóricas encontradas em projetos de pesquisa funcionam para realizar ações típicas na comunidade analisada.

Para a análise propriamente dita, embasamo-nos, essencialmente, em Swales (1990, 2004), e, mais precisamente no que o autor chama de passo retórico. O passo é entendido como fragmentos de texto que, ao serem combinados, compõem o movimento retórico ${ }^{1}$ e funcionam para que a função retórica do movimento seja realizada (MORENO; SWALES, 2018). Dessa forma, os passos são unidades menores dentro do texto que também possuem uma função comunicativa a ser realizada.

É recorrente que pesquisas sobre passos retóricos identifiquem também os movimentos retóricos que compõem a organização retórica ${ }^{2}$ dos gêneros (BIASIRODRIGUES, 2009; HENDGES, 2001; BEZERRA, 2002; COSTA, 2015; JUCÁ, 2006; MONTEIRO, 2016; OLIVEIRA, 2016). Tais pesquisas se baseiam em modelos de pesquisas que analisaram o mesmo gênero ou um gênero situado na mesma esfera de atividade, como é o caso de pesquisas como a de Jucá (2006), a qual utilizou o modelo CARS (Create a Research Space) (SWALES, 1990) para analisar as justificativas de projetos de pesquisa. A análise dos movimentos, embora mais difícil de ser operacionalizada, é importante para caracterizar valores de grandes comunidades como é caso da comunidade acadêmica. Por exemplo, o movimento "Estabelecendo um território de pesquisa" (SWALES, 1981, 1990) se mostrou bastante importante para caracterizar uma estratégia retórica consensual na grande maioria das culturas disciplinares.

Contudo, para analisar culturas disciplinares mais específicas e para lidar com amostras de textos pequenas provenientes de grupos locais, a análise de movimentos retóricos é bem menos adequada. Por esta razão, seguiremos outra proposta metodológica que visa não mais buscar a proposição de uma organização retórica dos gêneros, mas identificar os passos retóricos que compõem o gênero ou uma seção específica de um gênero (ALVES FILHO, 2018; ALVES FILHO; RIO LIMA, 2019). Esses trabalhos defendem que os passos retóricos são aquilo que se acha mais concretamente nos textos analisados, enquanto que o movimento retórico e, consequentemente, a apresentação de uma organização retórica dependem de "fatores extralinguísticos como propósitos comunicativos, interações e processos cognitivos" (ALVES FILHO, 2018, p. 138), difíceis de serem aprendidos no tipo de pesquisa aqui empreendida. A dificuldade surge em razão da multiplicidade de propósitos comunicativos que podem ser identificados em um mesmo corpus ao ser investigado por analistas diferentes, pois os movimentos podem ser nomeados diferentemente e pode-se chegar a outros propósitos que não estavam explícitos inicialmente (ASKEHAVE; SWALES, 2009).

\footnotetext{
1 "A 'move' $[\ldots]$ is a discoursal or rhetorical unit that performs a coherent communicative function in a written or spoken discourse.” (SWALES, 2004, p. 228).

2 "estrutura de composição textual regular e padronizada para se configurar como um gênero" (HEMAIS; BIASIRODRIGUES, 2005, p. 122).
} 
Há, ainda, uma terceira via metodológica proposta por Lim (2012), a qual buscou compreender como se estabelece o nicho (SWALES, 1990) em introduções de artigos de pesquisa da área de Administração por meio da análise de passos retóricos específicos e já identificados em outras pesquisas. Assim, parte-se de um modelo prévio e abandona-se a postulação de uma organização retórica. Ademais, o trabalho de Lim (2012) mostra que os passos retóricos podem se realizar de diversas maneiras, no caso a indicação de lacunas ocorre por meio de quatro caracterizações diferentes.

Compreendendo as possibilidades metodológicas, iremos focar a investigação nos passos e não nos movimentos nem na organização retórica, tendo em vista a potencial objetividade maior na identificação dos passos. Neste sentido, seguimos a tradição pedagógica da área de Inglês para Fins Específicos no que se refere ao seu objetivo de facilitar a aprendizagem da escrita de determinado gênero por escritores iniciantes.

Neste artigo, analisamos os seguintes passos retóricos "Relatando pesquisa prévia"; "Indicando lacuna de pesquisa"; e "Indicando problemas do mundo real"; identificados por Alves Filho (2018) em seções de justificativa de projetos de pesquisa de linguística. Essa escolha se deu em função da necessidade de se conhecer a área de pesquisa e os trabalhos que nela são desenvolvidos, como também indicar o espaço que a pesquisa vai ocupar (GIL, 2002; MOTTA-ROTH; HENDGES, 2010; PRODONOV; FREITAS, 2013). Em segundo lugar, pesquisas anteriores sobre a organização retórica do projeto de pesquisa ou sobre seções do projeto mostram resultados diferentes entre a utilização desses passos em projetos escritos por experts e projetos escritos por pesquisadores iniciantes.

A investigação de Connor e Mauranen (1999), a qual analisou projetos de pesquisa escritos por pesquisadores renomados e experientes em suas áreas de pesquisa e submetidos a uma agência de fomento da União Europeia, mostrou a presença de indicação de lacunas (chamado pelas autoras de movimentos), e que essas lacunas eram relacionadas tanto aos aspectos teórico-metodológicos quanto ao mundo social. Evidenciaram também a presença do Movimento "Reportando pesquisa prévia" como necessária à proposição de uma pesquisa.

Investigações sobre a fundamentação teórica (ALVES FILHO; RIO LIMA, 2019) e a sobre a justificativa (JUCÁ, 2006; SILVA, 2015; ALVES FILHO, 2018) de projetos de pesquisa submetidos a uma seleção de mestrado ou desenvolvidos durante o mestrado, portanto com pesquisadores em início de carreira, identificaram a escassez do relato de pesquisas prévias e das lacunas de pesquisa. $\mathrm{Na}$ Linguística, a área mais investigada, passos que relatam pesquisa prévia e, também, passos para indicar lacunas de pesquisa ocorreram com menos frequência do que aqueles que abordavam conceitos fundamentais e que inseriam a pesquisa em um território de pesquisa, sem estabelecer o seu nicho.

Considerando, portanto: (i) que o gênero projeto de pesquisa é um gênero de bastidores ou occluded genre (SWALES, 2004); (ii) que há poucas pesquisas sobre ele em contexto brasileiro; (iii) que essas pesquisas se concentram principalmente na escrita de pesquisadores iniciantes; (iv) que há diferenças na utilização dos passos retóricos de relato de pesquisas prévias e de indicação de lacuna em projetos de iniciantes e de experts; analisamos projetos de pesquisa escritos por experts e investigamos sobre os passos "Relatando pesquisa prévia", "Indicando lacuna de pesquisa" e "Indicando problemas do mundo real", objetivando, com isso, compreender um pouco mais sobre o funcionamento do gênero projeto de pesquisa.

O corpus é composto por 12 (doze) projetos da área de Química, os quais foram analisados considerando todo seu texto e não uma seção específica, escritos por pesquisadores experientes e que foram submetidos a editais de bolsa de produtividade e do programa de iniciação científica (PIBIC). O projeto de pesquisa é um gênero de bastidores, que não possui publicação, consequentemente, a única maneira de adquiri-los é entrar em contato diretamente 
com os pesquisadores e pedir sua liberação. É por essa razão também que o corpus é parcialmente heterogêneo, havendo projetos de bolsa de produtividade e de PIBIC.

Entretanto, o perfil dos autores dos projetos confere homogeneidade mínima que não compromete os resultados da investigação, uma vez que esses pesquisadores já se aculturaram (BERKENKOTTER; HUCKIN, 1995), por sua constante participação na comunidade discursiva realizando pesquisas e divulgando-as por meio de diversos gêneros acadêmicos. De fato, embora compreendamos que a situação retórica se mostra um tanto diferente, ao analisarmos passos retóricos específicos, é possível encontrar estratégias que permeiam o gênero projeto de pesquisa na área de Química em qualquer momento, haja vista serem escritos por pesquisadores experientes e conhecedores dos valores e crenças de suas comunidades discursivas e serem avaliados por pesquisadores experientes de mesmo perfil acadêmico.

Feitas essas considerações, apresentaram-se as seguintes indagações: os pesquisadores experientes da área de Química recorrem a esses três passos retóricos para construir a relevância da sua proposta de pesquisa? Se recorrem a esses passos, como eles são realizados? Há relação entre eles no interior dos textos, de modo que um passo contribua para a construção do outro? E foram estabelecidos estes objetivos: a) identificar se há ou não recorrência dos passos "Relatando pesquisa prévia", "Indicando lacuna de pesquisa" e "Indicando problemas do mundo real"; b) descrever e caracterizar as realizações desses passos retóricos; e c) verificar se na escrita do projeto eles se relacionam entre si.

Analisar as estratégias retóricas de uma área específica permite evidenciar convenções genéricas valorizadas na escrita dos gêneros que circulam naquela comunidade discursiva particular (TARDY, 2003), auxiliando na compreensão dos conhecimentos genéricos ou tipificados sobre como responder adequadamente às situações retóricas (MILLER, 2012[1984]).

\section{ESTRATÉGIAS RETÓRICAS PARA INDICAR A RELEVÂNCIA DE UMA PESQUISA}

Uma das funções retóricas conhecidas do projeto de pesquisa (doravante $\mathrm{PdP}$ ) em diversos contextos é persuadir seu leitor da relevância da pesquisa para os fins que lhe são específicos (CONNOR; MAURANEN, 1999), resultado de sua produção em situações de avaliação. Esse é o caso da avaliação dos projetos para bolsa de produtividade e de PIBIC indicar ou não a concessão de bolsas.

Há um entendimento consensual de que a relevância da pesquisa emerge da capacidade do pesquisador de escolher um tema que seja, simultaneamente, interessante para ele, mas que se harmonize também com as demandas da área e das instituições em que se insere (BARROS, 2005). Ou seja, a escolha do tema depende da capacidade do pesquisador de compreender o projeto como um espaço de tensões ou de lutas (TSENG, 2011). Isso porque o projeto constróise em situações retóricas de adequação ao próprio interesse do pesquisador, aos objetivos da instituição e à pressão dos pares que atuam na mesma área.

Os pontos de tensão podem ser superados por meio da seleção de bibliografia relevante para aquele tema, a fim de que se possa descobrir o que os pesquisadores já fizeram e estão fazendo atualmente na área (MOTTA-ROTH; HENGDES, 2010). Esse processo envolve conhecer teorias vigentes e relevantes e aderir ao que é considerado, pelas áreas, como relevante para ser pesquisado, em termos de estabelecimento de um problema, fundamentação teórica e proposição de procedimentos metodológicos que irão permitir alcançar os objetivos da pesquisa (PRODONOV; FREITAS, 2013). 
Assim, o acesso a referências teóricas e àquilo que chamamos de pesquisas prévias ${ }^{3}$ é colocado como solução para diminuir as tensões no momento de escrita do PdP e para estabelecer a atualidade e, consequentemente, a relevância de uma pesquisa. Em face disso, consideramos relevante compreender estratégia retórica "Relatando pesquisa prévia" (doravante RPP), uma vez que tal compreensão pode orientar a escrita de pesquisadores das diversas áreas, evidenciando valores que seriam gerais a uma proposta de pesquisa. Consequentemente, por sua generalização, demonstra-se também a importância de pesquisas focadas em comunidades discursivas específicas.

A decisão de não investigar os passos que abordam as referências teóricas (por meio de definição ou explicação de conceitos; de inserção em uma área de pesquisa; de discussões teóricas em geral) se justifica porque tais referências são utilizadas para estabelecer o quadro teórico da pesquisa (BARROS, 2005). Em contrapartida, analisar o relato de pesquisas prévias permite descobrir de que maneira os pesquisadores se apropriam da metodologia, da análise, das discussões e das conclusões das pesquisas anteriores para reivindicar a relevância da pesquisa.

Por outro lado, um PdP é visto como a proposição de algo novo e não só um relato de pesquisas prévias, já que seu objetivo não é investigar aquilo que já foi estudado e para o qual já se tem resposta (BARROS, 2005). É por isso que os pesquisadores em geral defendem seu projeto por meio do confronto ou discussão entre as referências teóricas e as pesquisas prévias (MOTTA-ROTH; HENDGES, 2010), com vistas a estabelecer um problema de pesquisa relevante. Essa discussão torna relevante a investigação do passo "Indicando lacuna de pesquisa" (doravante ILP), por meio do qual o pesquisador estabelece lacunas ou insuficiências na área, as quais carecem de resolução. Em suma, há a expectativa de que o pesquisador precisa se inserir em uma área de pesquisa, mas precisa também revelar lacunas nos trabalhos prévios (CONNOR; MAURANEN, 1999).

É relevante questionar como a lacuna de pesquisa é estabelecida, considerando que as pesquisas prévias possuem diversos aspectos que podem ser apontados como insuficientes (metodologia, resultados etc.) ou sequer foram realizadas ainda. Lim (2012) propõe quatro realizações da indicação de lacuna: (i) ausência total de pesquisas prévias; (ii) pesquisa insuficiente; (iii) limitação em pesquisa prévia; ou (iv) descobertas conflitantes em pesquisas prévias. Tal proposta será tomada como base para as análises do passo ILP, tendo em vista o seu potencial heurístico.

Compreendemos que o gênero analisado por Lim (2012) foi o artigo de pesquisa, no entanto, a introdução de artigos é considerada como semelhante ao projeto de pesquisa por sua necessidade de estabelecer um território, apresentar um problema ou lacuna e mostrar como o estudo irá responder à pergunta e preencher a lacuna (CONNOR; MAURANEN, 1999). Ademais, ao olharmos a cadeia de gênero (SWALES, 2004), vemos que normalmente o artigo de pesquisa é produzido após a realização de uma pesquisa que foi inicialmente pensada e proposta por um PdP. Por isso, ambos os gêneros guardam semelhanças acerca das informações que apresentam e das estratégias retóricas que os realizam.

Por fim, Connor e Mauranen (1999) descobriram que os PdP podem expor lacunas que indicam problemas ou limitações que são encontrados no mundo real e social, e que serão sanados por meio da pesquisa proposta no PdP. Por essa razão, decidimos investigar o passo

\footnotetext{
${ }^{3}$ As referências teóricas são os textos apropriados pelos escritores dos PdP para definir conceitos e estabelecer o quadro teórico da pesquisa. As pesquisas prévias são as publicações já realizadas sobre o tema da pesquisa, nas quais há a definição de um problema de pesquisa, de objetivos e de metodologia e há também a apresentação de resultados e conclusões após a realização de análises (RIO LIMA, 2019).
} 
"Indicando problemas do mundo real" (doravante IPMR), tendo em vista que ele também permite ao pesquisador argumentar a favor a relevância da pesquisa.

\section{3 "RELATANDO PESQUISA PRÉVIA": FOCO EM PESQUISAS PUBLICADAS EM ARTIGOS}

No levantamento quantitativo, há alta recorrência do passo retórico RPP, o qual ocorreu em todos os doze projetos analisados e, em nove PdPs, esse passo ocorreu várias vezes ao longo do texto. Esses passos foram identificados com base nos verbos da revisão de literatura indicados por Motta-Roth e Hendges (2010).

Para relatar pesquisas prévias, são apresentados, predominantemente, aspectos metodológicos (identificados por verbos de procedimentos de análise, como testar, avaliar, conduzir, usar/utilizar, sintetizar etc.) e resultados de investigações prévias (por meio de verbos como mostrar, demonstrar, fornecer, resultar, encontrar, produzir, desenvolver etc.), mas há relatos de objetivos (analisar, investigar, comparar dentro outros verbos predominantemente utilizados no infinitivo), de temas e de conclusões (por meio de verbos como propor, concluir, sugerir, considerar etc.). Observamos também se havia ou não referências bibliográficas no texto apoiando o relato das pesquisas prévias (uso de expressões como estudos, pesquisa e a própria apresentação da referência). As marcas linguísticas de identificação dessas características estão destacadas em negrito nos exemplos.

(Q1) A variabilidade da composição quiral de terpenóides foi realizada pelo estudo de Castillo e colaboradores [57] [PESQUISA PRÉVIA REFERENCIADA] analisando [OBJETIVO] os óleos essenciais de Mentha piperita, no qual foram relacionados [PROCEDIMENTO METODOLÓGICO] à constituição enantiomérica com diferentes origens da planta, na Espanha. Como resultado deste estudo, realizado [PROCEDIMENTO METODOLÓGICO] com o uso de duas fases estacionárias quirais derivadas de ciclodextrinas, foram encontradas [RESULTADOS] pequenas variações na distribuição enantiomérica dos terpenos quirais bioativos. Concluindo [CONCLUSÕES], os autores consideraram [CONCLUSÕES] que a variação enantiomérica de terpenos em Mentha piperita, é independente da origem geográfica da planta na Espanha.

(Q2) Olajide et al. (2013) [PESQUISA PRÉVIA REFERENCIADA] mostraram [RESULTADOS] que a atividade anti-inflamatória das cascas do caule de A. occidentale observada em cultura de células BV-2 expostas à LPS se deve à redução na produção de NO, PGE2, TNF- $\alpha$, e interleucina 6 e à inibição das enzimas COX2 NO induzível, provavelmente via inibição na via dos fatos MAPK e NF-kB.

(Q3) Recentemente, nossa equipe produziu [RESULTADOS] um filme baseado na blenda do polissacarídeo de goma de cajueiro e álcool polivinílico (CGP/PVA). O filme polimérico apresentou [RESULTADOS] características bastante interessantes, sendo utilizado como suporte para imobilização de enzimas quitinolíticas, permitindo propor sua aplicação como material bioativo a ser utilizado em embalagens (Silva et al., 2012) [PESQUISA PRÉVIA REFERENCIADA].

Os exemplos acima ilustram três modos de realizar o RPP: a) fazendo uma síntese dos diversos aspectos da pesquisa citada (objeto de estudo, procedimentos metodológicos, resultados e conclusões) - caso de Q1; b) concentrando-se em um único aspecto de uma única 
pesquisa de outrem - caso de Q2; c) relatando pesquisa prévia do próprio autor do projeto, objetivando mostrar que o estudo anterior obteve resultados satisfatórios e que não há repetição de pesquisa com o novo projeto - caso de Q3.

A alta recorrência de RPP permite ainda uma análise quantitativa complementar sobre os tipos de referências bibliográficas (livro, capítulo de livro, artigo de pesquisa, tese, dissertação e monografia) mencionadas no projeto, pois mostra quais gêneros estão sendo mais lidos e valorizados para a proposição de uma nova pesquisa. A presença predominante de artigos de pesquisa nas referências sugere a valorização de referências que são frutos de investigação e que foram publicadas recentemente em periódicos da área (Tabela 1).

Tabela 1 - Tipos de referências

\begin{tabular}{cccccc}
\multicolumn{2}{c}{ Referências teóricas } & \multicolumn{5}{c}{ Pesquisas prévias } \\
\hline Livro & Capítulo de livro & Artigo de pesquisa & Tese & Dissertação & Monografia \\
\hline 23 & 7 & 472 & 8 & 6 & 0 \\
\hline
\end{tabular}

Fonte: elaborado pelos autores.

A presença desse passo em todos os projetos confirma expectativas em um PdP por parte dos manuais de escrita acadêmica (GIL, 2002; MOTTA-ROTH; HENDGES, 2010; PRODONOV; FREITAS, 2013) e de investigações empíricas (CONNOR E MAURANEN, 1999) ao mostrar que os experts evidenciam seu conhecimento prévio sobre a área e expõem as informações que são mais relevantes sobre cada estudo (pois nem sempre comentam todos os aspectos das pesquisas prévias) e que são importantes para construção da relevância da pesquisa.

\section{4 "INDICANDO LACUNA DE PESQUISA": FOCO EM AUSÊNCIA, INSUFICIÊNCIA E LIMITAÇÕES METODOLÓGICAS, MAS SEM CONFRONTO DIRETO}

A frequência do passo ILP foi mediana, pois ocorreu em seis dos doze projetos e ocorreu somente nos projetos de bolsa de produtividade. Essa presença somente em PdPs de bolsa de produtividade pode ser pensada através da finalidade dessa bolsa, qual seja, "valorizar pesquisadores que possuam produção científica, tecnológica e de inovação de destaque em suas respectivas áreas do conhecimento e incentivar o aumento da produção científica, tecnológica e de inovação de qualidade" (CNPQ, 2019). Há uma expectativa da agência de que pesquisadores saibam expor as inovações da pesquisa, as quais podem ser expostas pela indicação de lacunas na área (BARROS, 2005; MOTTA-ROTH; HENDGES, 2010).

No corpus, das quatro estratégias observadas por Lim (2012), as duas mais usadas foram: destacar pesquisa insuficiente em um aspecto específico e revelar uma limitação em pesquisa prévia (Tabela 2).

Tabela 2 - Recorrência da realização de ILP

\begin{tabular}{ccccc}
\hline & $\begin{array}{c}\text { Ausência total } \\
\text { de pesquisas }\end{array}$ & $\begin{array}{c}\text { Pesquisa } \\
\text { insuficiente }\end{array}$ & $\begin{array}{c}\text { Limitação em } \\
\text { pesquisa prévia }\end{array}$ & $\begin{array}{c}\text { Descobertas } \\
\text { conflitantes }\end{array}$ \\
\hline Total de ocorrências & 5 & 15 & 10 & 1 \\
\hline Total de projetos & $3 / 6$ & $5 / 6$ & $5 / 6$ & $1 / 6$
\end{tabular}

Fonte: elaborado pelos autores. 
Os dados da Tabela 2 sugerem uma valorização de PdPs que sejam capazes de propor trabalhos com objetos de estudos, temas e substâncias que não foram investigados ou que foram pouco investigados na área. Isso indica a relevância do ineditismo da pesquisa. No entanto, é notória a quase ausência de críticas ou confrontos diretos entre pesquisas prévias (MOTTAROTH E HENDGES, 2010).

Para indicar ausência total de pesquisas prévias sobre uma determinada característica, os pesquisadores focaram em três aspectos: objeto de estudo (Q7), falta de aplicação de uma substância em um determinado contexto metodológico e inexistência de patentes de uma substância específica (Q8), por meio de marcas linguísticas como: nenhum, nunca, inexplorado, ineditismo, sem etc.

(Q7) Desta vez deverão ser investigadas as espécies Sinningia conspícua, S. gerdtiana, S. hatschbachii, S. macrostachya, S. mauroana e S. reitzii, que ocorrem naturalmente no estado do Paraná, e nunca [AUSÊECIA TOTAL] tiveram qualquer estudo químico ou biológico.

(Q8) Com base na leitura das reivindicações das patentes acima referidas, verificou-se que nenhum [AUSÊENCIA TOTAL] dos pedidos de patentes se refere ao desenvolvimento de um inibidor de corrosão específico para o biodiesel à base de amidas graxas, o que mostra o ineditismo do presente trabalho [AUSÊENCIA TOTAL] considerando que a eficiência do inibidor de corrosão depende também do meio corrosivo.

A recorrência maior de indicações de lacunas se dá em relação a pesquisas insuficientes. Essa maior frequência levanta o questionamento de porque é difícil afirmar categoricamente a ausência total de pesquisa. A resposta relaciona-se à dificuldade de se identificar todos os trabalhos sendo realizados no mundo inteiro, o que aponta para a cautela dos pesquisadores indicarem lacunas de pesquisa.

Já a insuficiência de pesquisas é demonstrada pela apresentação de aspectos pouco investigados (Q9) e pela apresentação da baixa porcentagem de estudos sobre um tópico ou procedimento metodológico (Q10). Assim, o pesquisador informa que algo ainda precisa ser feito e que o projeto proposto irá resolver. Esse passo foi encontrado por algumas dessas marcas linguísticas: pouco, apenas, não, ainda, incipiente, pequeno, restrito, falta etc.

(Q9) O processo de exsudação, embora comum, ainda não é bem conhecido [INSUFICI $\hat{E} \boldsymbol{N C I A}]$ em seus aspectos bioquímicos.

(Q10) Estes estudos representam uma pequena [INSUFICIÊECIA] proporção de temas que envolvem a CG enantiosseletiva como ferramenta de análise. Apesar disto, um levantamento feito em 2005, estimou que com óleos essenciais, apenas 4\% [INSUFICI $\hat{E} N C I A]$ das publicações fazem o uso da cromatografia quiral [103] [PESQUISA PRÉVIA REFERENCIADA].

Para "revelar uma limitação em pesquisa prévia", o foco ocorreu apenas nas limitações metodológicas (pelo uso de marcas linguísticas como porém, insuficiente, dificil, tóxico, menor, complicado, inconveniente, desvantagem etc.), revelando a valorização da importância de um olhar crítico para as metodologias dos estudos prévios, para que seja possível propor novos caminhos que solucionem esses problemas (Q11 e Q12).

(Q11) A rota mais conhecida e consolidada é a metílica, porem, alguns fatores são citados

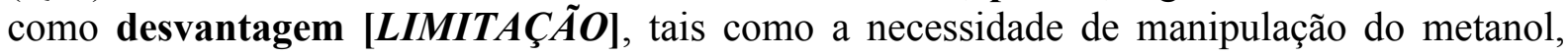




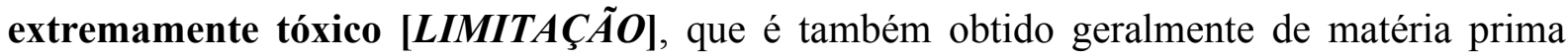
fóssil.

(Q12) Além disso, nestes estudos [sobre biodiesel] [PESQUISAS PRÉVIAS NÃO REFERENCIADAS] o efeito da corrosão é analisado somente [LIMITAÇÃO ] visualmente

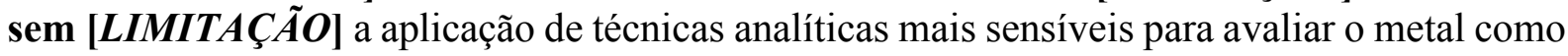
o método gravimétrico e a espectrofotometria de absorção atômica que são propostos no presente trabalho.

Contrastar descobertas conflitantes em pesquisas prévias foi a realização com menos ocorrência e não ocorreu da maneira como Lim (2012) descreve. Essa diferença na realização pode ser explicada pelas diferenças das áreas investigadas e pela diferença dos gêneros. No PdP de Química, não são contrastados resultados de pesquisas prévias, mas posicionamentos diferentes. Consequentemente, esses posicionamentos levam os pesquisadores a proporem pesquisas que possam resolver tais impasses existentes. Por essa razão propomos uma nomeação diferente a essa realização: "contrastando posicionamentos conflitantes dentro da área".

No excerto (Q13), há pesquisadores que defendem a criação de biodiesel por meio de uso de óleos comestíveis e outros que defendem o uso de óleos não comestíveis. Se há esse conflito (identificado pelo termo conflito e pela exposição de ideias diferentes), significa que ainda há espaço para novas pesquisas. Assim, o pesquisador localiza sua proposta nessa lacuna de pesquisa gerada por posições divergentes.

(Q13) Existe hoje, um conflito [POSICIONAMENTO CONFLITANTE] entre pesquisadores defensores de óleos comestíveis e não comestíveis (edible and non-edilbe oils). Este conflito tem suporte nos usos de óleos não comestíveis como matéria prima para a síntese de biocombustiveis (biodiesel) [3] [PESQUISA PRÉVIA REFERENCIADA].

Outra característica de ILP é que, apesar de haver implicitamente (patentes, p. ex.) e explicitamente (citação por números, p. ex.) a presença de referências a estudos prévios, o próprio escritor do PdP é quem atribui e indica quais as lacunas da área e não os autores dos estudos por ele referidos. Isso se explica pela expectativa de que o autor do PdP, após acessar a literatura, seja capaz de, ele mesmo, apontar o que precisa ser feito. De fato, utilizar outros autores para estabelecer uma lacuna pode ser visto como menos inovador, por isso a necessidade de o próprio pesquisador mostrar sua capacidade crítica e autoral de indicar uma lacuna, o que ocorreu principalmente em PdPs para bolsa de produtividade.

\section{5 "INDICANDO PROBLEMAS DO MUNDO REAL": VIÍNCULOS COM A ÁREA DE PESQUISA E COM A SOCIEDADE EM GERAL}

O passo IPMR foi encontrado em onze dos doze projetos, o que indica sua alta relevância na amostra analisada.

O mundo real que nomeia o passo IPMR deve ser entendido como o mundo da vida prática (ALVES FILHO, 2018) ou o mundo social no qual a pesquisa proposta está inserida. Além disso, o problema aqui não deve ser confundido com as indagações ou perguntas de pesquisa existentes no projeto, as quais se apresentam em forma de interrogação (GIL, 2002). Portanto, quando se diz "problemas do mundo real", consideram-se as dificuldades, as 
adversidades, bem como as crises que ocorrem na sociedade e que precisam e podem ser solucionadas por meio de uma pesquisa.

Quando se observa a mediação ou a ausência de mediação de leitura, esse passo se realiza de três maneiras: mediação de leitura por meio de pesquisas prévias ou por meio de documentos governamentais, dados estatísticos públicos e ausência de mediação de leitura.

A apresentação de um problema do mundo real através de pesquisas prévias (Q14) sugere a relevância do problema dentro da própria área de pesquisa, pois traz comentários acerca dos resultados ou conclusões de pesquisas prévias e demonstra que: 1) o problema já foi investigado, mas ainda não foram respondidas todas as indagações acerca dele; 2) o fato de já haver outros pesquisadores investigando esse problema do mundo real é um argumento para mostrar a preocupação em resolver problemas que são atuais e que possuem relevância social e científica. Assim, por meio desse passo retórico, a pesquisa consegue lidar com as pressões institucionais e dos pares presentes durante a escrita do PdP (BARROS, 2005; TSENG, 2011).

(Q14) Entretanto existem algumas limitações [PROBLEMA] na atual terapêutica, como a ineficácia [PROBLEMA] na prevenção e reincidência de úlceras em usuários de $\mathrm{DAINE}^{\mathrm{ee}} \mathrm{s}$, diminuição na eficácia de erradicação de $H$. pylori e em úlceras não associadas a $H$. pylori ou DAINE"es (Yuan et al., 2006)

Por outro lado, quando o problema do mundo real é mediado pela leitura de documentos oficiais ou por dados públicos, o pesquisador enfatiza a sua relevância para a sociedade em geral. Em Q15, na nota de rodapé "10", tem-se um problema que foi percebido por uma análise feita na Câmara dos Deputados e está sendo investigado pelos legisladores do país, o que indica sua importância para a sociedade.

(Q15) O fenômeno de deposição orgânica também é um dos desafios [PROBLEMA] operacionais existentes ao longo da produção de petróleo. ${ }^{10}$ A deposição orgânica obstrui [PROBLEMA] as tubulações de produção de óleo, diminuindo [PROBLEMA] assim o escoamento do fluido.

A ausência total de mediação de leitura ocorre quando o autor indica o problema do mundo real sem citar referências de pesquisas prévias ou de documentos. Essa indicação parte de um conhecimento de mundo do próprio pesquisador, embora não possamos afirmar ser um problema vivenciado pessoalmente. Ao contrário das ocorrências anteriormente citadas, quando há a ausência de fontes que embasam o problema do mundo real, tem-se uma busca para acessar um conhecimento compartilhado de mundo entre o pesquisador e o leitor, pois são informações que podem ser encontradas em veículos midiáticos públicos, como jornais. É o caso do exemplo Q16.

(Q16) Diversos esforços preventivos, como o controle do inseto vetor ou a vacinação contra Leishmania foram aplicados para combater a leishmaniose, mas essas medidas não têm sido bem sucedidas [PROBLEMA], em sua totalidade.

No excerto (Q16), os problemas causados pela falta de controle de um vetor e da vacinação são bastante divulgados, já que, para vacinas, sempre existem campanhas de vacinação públicas nos meios midiáticos. Reiteramos que, ao apresentar problemas sem mediação de leitura, o escritor apresenta informações que são do conhecimento compartilhado e que não precisam de validação por meio de citações. 
A identificação de IPMR se baseou em marcas linguísticas que indicam problemas: obstruir, diminuir, preocupar, problema, problemática, dificuldade, limitações, ineficácia, desafio, preocupante, indesejável etc. Das três realizações investigadas, a mais frequente foi a indicação de problemas do mundo real sem mediação de leitura e, em seguida, a mediação com o uso de pesquisas prévias. Esses aspectos evidenciam a relevância do PdP tanto para a sociedade como para a área de estudo.

\section{AS RELAÇÕES QUE OS PASSOS MANTÊM ENTRE SI E COM A PROPOSIÇÃO DO PROJETO}

Há uma visão bastante consensual de que a relevância de um projeto surge da conexão entre um amplo conhecimento da literatura da área e a capacidade de encontrar limitações nessa literatura (CONNOR; MAURANEN, 1999; BARROS, 2005; MOTTA-ROTH; HENDGES, 2010; PRODONOV; FREITAS, 2013). Com base nesta assunção, partimos da hipótese de que para a construção da relevância do projeto os passos retóricos RPP e ILP estariam potencialmente inter-relacionados, aparecendo textualmente próximos entre si. A relação direta, neste caso, é a proximidade dos passos que ocorrem sequencialmente um após o outro, de maneira que uma informação complementa a outra, tecendo uma teia de informação que dão sentido e criam a relevância do projeto. Contudo, as descobertas revelaram que somente dois dos seis projetos que realizaram o passo ILP apresentaram uma relação direta entre essas duas estratégias.

Quando o RPP vem primeiro, ele contextualiza algum aspecto da pesquisa, evidenciando que algo já foi pesquisado e é conhecido. Na sequência, a indicação de lacuna mostra que há espaço para mais investigações. Nestes casos, a lacuna, normalmente, é relacionada a limitações metodológicas das pesquisas prévias. No caso em que a lacuna aparece antes do relato, há ênfase na insuficiência de pesquisas sobre um tópico, mostrando, em seguida, o pouco que já foi feito. A ordem e a relação entre esses passos estão relacionadas à lacuna que está sendo indicada.

Nos outros casos, temos o passo IPMR, seguido de ILP, para indicação de conflitos na área. Isto é, existe um problema que faz parte do mundo social, mas que ainda não foi resolvido teórico-metodologicamente porque não há consenso sobre a utilização de determinados produtos. Nesse contexto, há relação direta entre os problemas da área e os problemas do mundo real, uma vez que as investigações não foram suficientes para resolver o problema. Essa relação entre IPMR e ILP serve ainda para mostrar que os estudos são insuficientes.

O passo RPP após IPMR permite relacionar os problemas e as insuficiências da área com problemas do mundo real e mostra a necessidade de mais pesquisas para sanar os problemas dentro da própria área, o que, por consequência, sanaria o problema do mundo real.

\section{CONSIDERAÇÕES FINAIS}

Com base nos resultados das análises, constatou-se que os três passos retóricos RPP, ILP e IPMR estão bastante presentes nos projetos de Química. Com isso, verifica-se que essas estratégias retóricas tendem a ser valorizadas dentro dessa área e permitem ao escritor do projeto estabelecer a relevância de sua pesquisa, confirmando indicações presentes em manuais de escrita acadêmica (BARROS, 2005; MOTTA-ROTH; HENDGES, 2010; PRODONOV; FREITAS, 2013).

Observou-se também a diversidade de realizações possíveis de cada um desses passos, os quais apresentam características distintas e destacam informações que são relevantes para o 
projeto. No caso da área de Química, em nosso corpus, a metodologia das pesquisas prévias foi bastante importante, uma vez que é discutida nos passos RPP e ILP. As análises sugerem que o papel da metodologia é bastante relevante e é algo a ser destacado no PdP, seja para mostrar que a metodologia funciona e já foi utilizada por outros pesquisadores, seja para mostrar que ela deve ser modificada em algum de seus aspectos.

O passo IPMR é a estratégia retórica que insere o PdP dentro de um contexto social, fora do mundo teórico-metodológico. A alta recorrência desse passo indica a importância de o pesquisador ter consciência de que a pesquisa dele não ficará restrita às discussões acadêmicas, mas que será capaz de atenuar problemas sociais. Isso está conectado com a visão de que a academia não deve estar distante do mundo social, fechada em si mesma e que as pesquisas realizadas em universidades devem oferecer retorno à sociedade, mesmo que a um grupo específico.

\section{REFERÊNCIAS}

ALVES FILHO, F. Como mestrandos agem retoricamente quando precisam justificar suas pesquisas. Revista Brasileira de Linguística Aplicada, v. 18, n. 1, p. 131-158, 2018.

ALVES FILHO, F.; RIO LIMA, C. A. C. A escrita da fundamentação teórica em projetos de pesquisa na área de linguística. In: PEREIRA, R. C. M. (Org.). Cultura disciplinar e epistemes: representações na escrita acadêmica. João Pessoa: Ideia, 2019. p. 247-274.

ASKEHAVE, I.; SWALES, J. Identificação de gênero e propósito comunicativo: um problema e uma possível solução. In: BEZERRA, B. G. et al. (Orgs.). Gêneros e sequências textuais. Recife: Edupe, 2009. p. 221-247.

BARROS, J. d'A.. Projeto de pesquisa em História: da escolha do tema ao quadro teórico. Petrópolis: Vozes, 2005.

BERKENKOTTER, C.; HUCKIN, T. N. Genre knowledge in disciplinary communication: cognition, culture, power. New Jersey: Lawrence Erlbaum Associates Inc., Publishers, 1995. p. $1-44$.

BEZERRA, B. G. A organização retórica de resenhas acadêmicas. Linguagem em (Dis)curso, Tubarão, v. 3, n. 1, p. 37-68, 2002.

BIASI-RODRIGUES, B. O gênero resumo: uma prática discursiva da comunidade acadêmica. In: BIASI-RODRIGUES, B.; ARAÚJO, J. C.; SOUSA, S. C. T. de (Orgs.). Gêneros textuais e comunidades discursivas: um diálogo com John Swales. Belo Horizonte: Autêntica Editora, 2009. P. 49-75.

CONNOR, U.; MAURANEN, A. Linguistic analysis of grant proposals: European Union research grant. English for Specific Purposes, v. 18, n. 1, p.47-62, 1999.

CONSELHO NACIONAL DE DESENVOLVIMENTO CIENTÍFICO E TECNOLÓGICO

(CNPQ). Chamada CNPq no 06/2019: bolsas de produtividade em pesquisa, 2019.

Disponível em: http://resultado.cnpq.br/4615070488360730. Acesso em: 14 de jun. 2020. 
COSTA, R. L. S. da. Culturas disciplinares e artigos acadêmicos experimentais: um estudo comparativo da descrição sociorretórica. 2015. 243 f. Dissertação (Mestrado Acadêmico em Linguística Aplicada) - Universidade Estadual do Ceará, Ceará, 2015.

GIL, A. C.. Como elaborar projetos de pesquisa. 4. ed. São Paulo: Atlas, 2002.

HEMAIS, B.; BIASI-RODRIGUES, B. A proposta de John M. Swales para o estudo de gêneros textuais. MEURER, J. L.; BONINI, A.; MOTTA-ROTH, D. (Orgs.). Gêneros: teorias, métodos e debates. São Paulo: Parábola Editorial, 2005. p. 108-129.

HENDGES, G. R. Novos contextos, novos gêneros: a revisão da literatura em artigos acadêmicos eletrônicos. 2001. 138 f. Dissertação (Mestrado em Letras) - Universidade Federal de Santa Maria, Rio Grande do Sul, 2001.

JUCÁ, D. A organização retórico-argumentativa da seção de justificativa do gênero textual projeto de dissertação. 2006. 109 f. Dissertação (Mestrado): Universidade Federal do Ceará, 2006.

LIM, J. M.-H. How do writers establish research niches? A genre-based investigation into management researchers rhetorical steps and linguistic mechanisms. Journal of English for Academic Purposes, v. 11, p. 229-245, 2012.

MEURER, J. L.; BONINI, A.; MOTTA-ROTH, D. (Orgs.). Gêneros: teorias, métodos e debates. São Paulo: Parábola Editorial, 2005.

MILLER, C. R. Estudos sobre gênero textual, agência e tecnologia. São Paulo: Parábola Editorial, 2012. p. 21-41.

MONTEIRO, B. N. Organização retórica e estruturação sequencial da seção de metodologia do gênero projeto de pesquisa. 2016. Dissertação (Mestrado em Letras) Universidade Federal do Piauí, Teresina, 2016.

MORENO, A. I.; SWALES, J. M. Strengthening move analysis methodology towards bridging the functionform gap. English for Specific Purposes, n. 50, p. 40-63, 2018.

MOTTA-ROTH, D; HENDGES, G. Produção textual na universidade. São Paulo: Parábola Editorial, 2010.

OLIVEIRA, F. V. A. Organização retórica da seção de considerações finais do gênero monografia em comunidades disciplinares distintas. 2016. Dissertação (Mestrado em Letras) - Universidade Federal do Piauí, Teresina, 2016.

PRODONOV, C. C.; FREITAS, E. C. de. Metodologia do trabalho científico: métodos e técnicas da pesquisa e do trabalho acadêmico. 2.ed. Novo Hamburgo: Feevale, 2013.

RIO LIMA, C. A. C. Estratégias retóricas do gênero projeto de pesquisa nas áreas de linguística e química: relatando pesquisas prévias, indicando lacuna de pesquisa e problemas do mundo real. 2019. 161 f. Dissertação (Mestrado em Letras) - Universidade Federal do Piauí, Teresina, 2019. 
SILVA, C. R. B. Movimentos retóricos da seção de justificativa de projetos de pesquisa da área de história. Revista Ininga, v. 2, n. 1, p. 33-53, 2015.

SWALES, J. M. Aspects of article introductions. Birmingham: The University of Aston, 1981.

SWALES, J. M. Genre analysis: English in academic and research settings. Cambridge: Cambridge University Press, 1990.

SWALES, J. M. Research genres: Explorations and Applications. Cambridge: Cambridge University Press, 2004.

SWALES, J. M. Reflections on the concept of discourse community. Asp, v. 69, mar., p.7-19, 2016.

TARDY, C. A genre system view of the Funding of Academic Research. Written Communication, v. 20, n. 1, jan., p. 7-36, 2003.

TSENG, M.-Y. The genre of research grant proposals: towards a cognitive-pragmatic analysis. Journal of Pragmatics, n. 43, p. 2254-22, 2011.

Recebido em: 18 de março de 2020

Aceito em: 18 de junho 2020

Publicado em Setembro de 2020 